\title{
Detection of Nanoparticles of Differing Composition for High Resolution Labeling
}

\author{
D.A. Meyer, J. Heintz, and R.M. Albrecht
}

Department of Animal Sciences, University of Wisconsin, 1675 Observatory Drive, Madison, WI 53706

Biological activity takes place in the context of multi-molecular complexes. An understanding of cellular function requires not only identification of the various molecular species but an understanding of their physical relationships. The use of high resolution labeling to provide molecular or sub-molecular spatial resolution is one approach to identification and localization of molecular species or even individual epitopes on a single molecule. Small colloidal metal particles in the 1 to $10 \mathrm{~nm}$ size range coupled to active fragments of antibodies or active fragments of ligands provide specific probes within the size range required. However it is often necessary to be able to label two or more antigens simultaneously. This is necessary to accurately determine spatial relationships and co-localization patterns of individual molecular species in multi-molecular complexes internally or on cell surfaces. Also, it is necessary for the understanding of the relationship of structure-associated molecules and their interaction with cytoskeletal elements.

At the level of resolution attainable in the light microscope, fluorescent dyes having different excitation and emission frequencies can be used for simultaneous multiple labeling. For molecular and submolecular levels of resolution, various types of electron microscopy are commonly employed; however, the ability to obtain spatial relationships at high resolution via simultaneous labeling has been difficult. The classical system for multiple labeling immuno-EM uses electron dense markers of different sizes, commonly made of $\mathrm{Au}$, which need to differ considerably in size to prevent overlapping of signals and to allow unambiguous allocation of labels to antigens. Comparison of results obtained with different sized markers is problematic, because larger markers, when bound to an antigen, may block more neighboring binding sites than smaller particles, or they can mask smaller labels thereby making them undetectable. Also, larger particles may bind to more than one epitope per bead, thus making it difficult to compare or quantify signal densities. Additionally, spatial resolution is substantially reduced with larger marker sizes.

We have utilized colloidal nanoparticles of the same size, to $3 \mathrm{~nm}$ diameter, but having differing elemental compositions or differing shapes as promising approaches to this goal[1,2]. Au, Ag, Pd, $\mathrm{Pt}, \mathrm{Ru} \mathrm{Rh}, \mathrm{Fe}$ and other colloidal nano-particles with uniform sizes can be used as labels. In some cases core-shell particles such as Au-coated Pt [3] can also be employed. In this case, each particle type is conjugated directly to a different primary antibody or antibody fragment. We have previously utilized ESI imaging of conjugates on ultrathin sections to identify the different particle types. For ESI, inelastically scattered electrons with element specific energy losses can be selectively used for imaging to generate elemental distribution maps. With this approach, labeling reactions can be compared directly, since labels are identified by their elemental composition. Also, the number of different reporters for multiple labeling immuno-EM is increased substantially in comparison to conventional techniques. Images of particles can be overlaid on zero loss TEM images providing a direct correlation between multiple specific labels and biological structure [3]. 
While the EELS imaging approach can be used effectively, it requires very thin specimens to limit interactions of the primary beam electrons with only one atom. The use of EDX to identify particle composition would be of advantage and permit imaging of thick sections which could be used for stereo pairs or for tomographic images. However, conventional EDX instrumentation lacked the resolution necessary to identify individual particles in the high resolution range. Recent advances in instrument and beam technology have substantially increased resolution attainable both in terms of particle size and mass required for an unambiguous signal. In the current study, we utilized an FEI TITAN 80-200 aberration corrected STEM coupled with an EDAX high resolution EDX detector. Initial studies utilizing colloidal nanoparticle labels of several different elemental compositions and sizes indicate that beam stability, size, and energy permit individual particles in the $3 \mathrm{~nm}$ range adjacent to one another to be readily identified and differentiated (figure 1). This provides the requisite resolution and detectability to perform high resolution simultaneous labeling on thick sections and whole mount preparations.

\section{References:}

1. D.A. Meyer et al., Micros. and Microanal. 12 (Suppl. 2) (2006) 32.

2. D.A. Meyer et al., Micros. and Microanal. 16 (2010) 33.

3. R. Bleher et al., J. Micros. 230 (2008) 388.
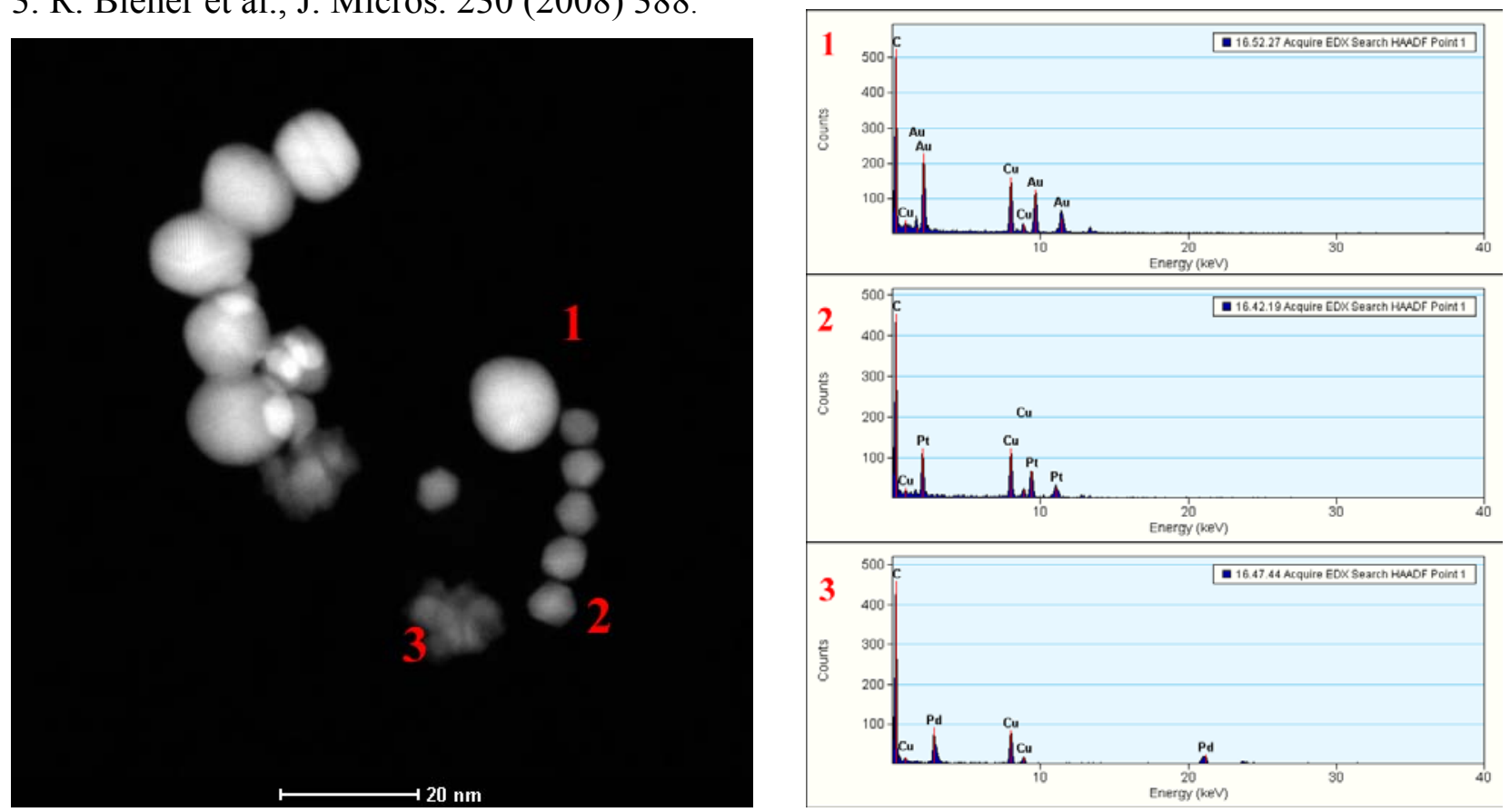

Figure 1. Scanning transmission electron micrograph of a specimen composed of cAu, cAg, cPd, and cPt particles, which can be conjugated to antibodies and other ligands useful for labeling, adsorbed onto a thin-film carbon support over copper grid. An ångstrom level probe was positioned over each of three particles as indicated in the micrograph, and EDX spectra were collected at each position for 200 seconds in order to identify the composition of each particle. The spectra indicate unambiguously that the particle at the first probe position is composed of $\mathrm{Au}$, the composition of the particle at the second position is $\mathrm{Pt}$, and at the third, Pd. EDX spectroscopy further indicated that no $\mathrm{cAg}$ particles were present in this field of view. Magnification $=1.8 \mathrm{MX}$, accelerating voltage $=200$ $\mathrm{keV}$, and tilt angle $=0$ degrees. 